\title{
Traveling interface modulations and anisotropic front propagation in ammonia oxidation over $\mathrm{Rh}(110)$
}

\author{
Matías Rafti, ${ }^{1,2}$ Benjamin Borkenhagen, ${ }^{3}$ Gerhard Lilienkamp, ${ }^{3}$ Florian Lovis, ${ }^{2}$ \\ Tim Smolinsky, ${ }^{2}$ and Ronald Imbihl $^{2, a}$ ) \\ ${ }^{1}$ Instituto de Investigaciones Fisicoquímicas Teóricas y Aplicadas (INIFTA), Fac. Cs. Exactas, \\ Universidad Nacional de La Plata, 64 y Diag. 113 (1900), La Plata, Argentina \\ ${ }^{2}$ Institut für Physikalische Chemie und Elektrochemie, Leibniz-Universität Hannover, Callinstr. 3-3a, \\ D-30167 Hannover, Germany \\ ${ }^{3}$ Institut für Energieforschung und Physikalische Technologien, Technische Universität Clausthal, Leibnizstr. 4, \\ 38678 Clausthal-Zellerfeld, Germany
}

(Received 22 July 2015; accepted 26 October 2015; published online 11 November 2015)

\begin{abstract}
The bistable $\mathrm{NH}_{3}+\mathrm{O}_{2}$ reaction over a $\mathrm{Rh}(110)$ surface was explored in the pressure range $10^{-6}-10^{-3}$ mbar and in the temperature range 300-900 K using photoemission electron microscopy and low energy electron microscopy as spatially resolving methods. We observed a history dependent anisotropy in front propagation, traveling interface modulations, transitions with secondary reaction fronts, and stationary island structures. (C) 2015 AIP Publishing LLC. [http://dx.doi.org/10.1063/1.4935274]
\end{abstract}

\section{INTRODUCTION}

The catalytic oxidation of ammonia with $\mathrm{O}_{2}$ on noble metals of the platinum group is of considerable interest due to the key role the Ostwald process plays in the chemical industry and due to the necessity of removing ammonia in exhaust gases. ${ }^{1,2}$ In addition, the $\mathrm{NH}_{3}+\mathrm{O}_{2}$ reaction was also shown to exhibit interesting non-linear behavior over $\mathrm{Rh}$ and $\mathrm{Pt}$ surfaces, i.e., rate oscillations and chemical wave patterns have been observed. ${ }^{3-9}$ The majority of the kinetic and mechanistic studies focus on the $\mathrm{NH}_{3}+\mathrm{O}_{2}$ reaction over $\mathrm{Pt}$ surfaces while comparatively few such investigations exist for Rh surfaces. ${ }^{10-12}$ The behavior of $\mathrm{Rh}$, however, is important as well because the $\mathrm{Pt} / \mathrm{Rh}$ gauze used in the Ostwald process contains up to $10 \%$ Rh. $^{1}$

At low pressure $\left(\mathrm{p}<10^{-3} \mathrm{mbar}\right)$, ammonia reacts with oxygen on $\mathrm{Pt}$ and $\mathrm{Rh}$ to yield as main products $\mathrm{N}_{2}, \mathrm{NO}$, and $\mathrm{H}_{2} \mathrm{O}$; at elevated pressure ( $\mathrm{p}>10^{-3}$ mbar), also $\mathrm{N}_{2} \mathrm{O}$ can be detected as product, $1,10,11$

$$
\begin{gathered}
4 \mathrm{NH}_{3}+3 \mathrm{O}_{2} \rightarrow 2 \mathrm{~N}_{2}+6 \mathrm{H}_{2} \mathrm{O}, \\
4 \mathrm{NH}_{3}+5 \mathrm{O}_{2} \rightarrow 4 \mathrm{NO}+6 \mathrm{H}_{2} \mathrm{O} .
\end{gathered}
$$

At lower temperature, $\mathrm{N}_{2}$ is the main product but with increasing temperature the selectivity shifts towards NO. Supported by quantum chemical calculations (density function theory), it was shown that adsorbed oxygen on $\mathrm{Pt}$ activates ammonia decomposition. ${ }^{11,13}$ The kinetics of ammonia decomposition was investigated on the various low-index single crystal planes of Rh. ${ }^{14}$ High temperature ammonia oxidation over a Rh wire was studied by Perez-Ramirez with the TAP (temporal analysis of products) technique. ${ }^{15}$

Ammonia oxidation over a $\mathrm{Rh}(110)$ surface was investigated in several low pressure ( $\mathrm{p}<10^{-3}$ mbar) studies. ${ }^{8,9,12}$ The interest in this particular reaction system stems from

\footnotetext{
a)E-mail: imbihl@pci.uni-hannvover.de.
}

the observation of rate oscillations and of a novel type of chemical wave pattern that was discovered in ammonia oxidation over $\mathrm{Rh}(110)$. When parameters were adjusted establishing equistability in the bistable regime of the reaction so that the interface between the two stable phases did not advance, some lateral small displacements (a few $\mu \mathrm{m}$ ) of the boundary propagated similar to a pulse along the interface. ${ }^{9}$ These localized excitations traveling along the interface of two surface phases have been reproduced with a general mathematical model but a mechanistic understanding is still missing. ${ }^{9}$ This novel type of chemical wave pattern has been brought in connection with the wave patterns which have been discovered in a closely related system, the $\mathrm{NO}+\mathrm{H}_{2}$ reaction over $\mathrm{Rh}(110) .{ }^{16-18}$ There, rectangular shaped wave patterns and travelling wave fragments were observed. Mechanistically, they were shown to be due to the large structural variability of the $\mathrm{Rh}(110)$ surface evidenced by a number of different $\mathrm{O}, \mathrm{N}$-induced reconstructions. Since the same atomic adsorbates, $\mathrm{N}$ and $\mathrm{O}$, are also present in the $\mathrm{NH}_{3}+\mathrm{O}_{2}$ reaction, it seemed reasonable to suspect that the basic excitation mechanism could be identical in both systems.

The primary motivation for this study has been to elucidate the mechanism for the traveling interface modulations. Photoemission electron microscopy (PEEM) with which the localized excitations have been studied yields no or only indirect structural and chemical information. For this reason, low energy electron microscopy (LEEM) is used to study the processes at the interface with higher spatial resolution and with the possibility to identify the local surface structure on a scale of micrometers with low energy electron diffraction ( $\mu$-LEED). The more detailed information provided by this study shows that the behavior of the system is far more complex than anticipated before. We observe a front anisotropy which depends on the direction of the transition, island formation in the submicrometer range, and transitions involving secondary reaction fronts. 


\section{EXPERIMENTAL}

The PEEM measurements are performed in a standard ultrahigh vacuum (UHV) system equipped with conventional low energy electron diffraction (LEED) optics and a differentially pumped quadrupole mass spectrometer (QMS) for rate measurements. The $\mathrm{Rh}(110)$ sample is prepared by repeated argon ion sputtering $\left(\mathrm{E}=1 \mathrm{keV}, \mathrm{p}(\mathrm{Ar})=2 \times 10^{-5} \mathrm{mbar}\right.$, and $\mathrm{t}=20 \mathrm{~min})$, oxidation treatments $\left(\mathrm{p}\left(\mathrm{O}_{2}\right)=3 \times 10^{-6}\right.$ mbar, $\mathrm{T}=750 \mathrm{~K}, \mathrm{t}=30 \mathrm{~min}$ ), and vacuum annealing cycles $(\mathrm{T}=1200 \mathrm{~K}, \mathrm{t}=1 \mathrm{~min})$ until a sharp LEED pattern is obtained. The surface cleanliness is checked via Auger electron spectroscopy. The sample is heated indirectly by a filament behind the backside of the crystal, either via radiation or via electron bombardment. Gases of purity 5.0 for oxygen and 2.5 for ammonia (both Linde AG) are used.

During the rate measurements, a cone of $\approx 2 \mathrm{~mm}$ opening, shielding a differentially pumped QMS, is brought $1 \mathrm{~mm}$ in front of the sample. In this way, detection of reaction products coming from the backside of the sample and from the filament is avoided. $\mathrm{NO}$ and $\mathrm{N}_{2}$ formation are followed via $\mathrm{m} / \mathrm{e}=30$ and $\mathrm{m} / \mathrm{e}=28 \mathrm{QMS}$ signals, respectively.

In PEEM (see, e.g., Ref. 19), UV light from a deuterium discharge lamp with maximum emission at $5.2-6.2 \mathrm{eV}$ photon energy is focused into a small spot on the sample $\left(\approx 2 \mathrm{~mm}^{2}\right)$. The emitted photoelectrons are collected by a magnifying electrostatic three-lens system, amplified by a channel plate and then imaged onto a phosphorous screen. The images are recorded with a CCD camera and digitized. PEEM images primarily the local work function but yields no direct information on the chemical identity or the structure of the adlayer. For this reason, complementary experiments are carried out in the facilities at Clausthal using $\mu$-LEED and LEEM. ${ }^{20}$ Using suitable illumination apertures, the electron diffraction patterns of small sample areas down to the $\mu \mathrm{m}$ range can be obtained ( $\mu$-LEED). In LEEM, specular or diffracted electrons are used to create image contrast of different adlayers on the surface with a lateral resolution down to $\sim 10 \mathrm{~nm}$.

\section{RESULTS}

An overview of the different experiments and of the different imaging techniques conducted in various $\mathrm{p}, \mathrm{T}$ regimes of the reaction is given in Table I. For technical reasons, LEEM is only feasible up to the $10^{-5}$ mbar range while the upper p-limit for PEEM measurements lies much higher, at $10^{-3}$ mbar.

\section{A. PEEM}

\section{Front anisotropy}

Essentially, two types of reconstructions exist in the $\mathrm{Rh}(110) / \mathrm{O}, \mathrm{N}$-system. ${ }^{21}$ Oxygen induces missing row type reconstructions $\mathrm{c}(2 \times 2 \mathrm{n})$ in which every $\mathrm{n}$-th $(\mathrm{n}=2,3,4,5)$ of the close-packed [110]-rows is missing whereas the $(2 \times 1)$ - $\mathrm{N}$ and $(3 \times 1)$ reconstructions of nitrogen are built up by $-\mathrm{Rh}-\mathrm{N}-\mathrm{Rh}$ - chains in the [001]-direction. The mixed coadsorbate structure $(2 \times 4)-2 \mathrm{O}, \mathrm{N}$ is formed by a $(1 \times 2)$ missing row reconstruction, i.e., it is oxygen which dictates the type of reconstruction. ${ }^{22}$ From the geometric corrugation, one could expect anisotropic front propagation either in the [110]-direction or in the [001]-direction depending on which type of reconstruction determines the spreading of a reaction front.

The $\mathrm{NH}_{3}+\mathrm{O}_{2}$ reaction exhibits bistability on $\mathrm{Rh}(110)$, i.e., transitions between two stable states occur via propagating reaction fronts. ${ }^{9}$ The PEEM images in Figs. 1 and 2 recorded at relatively high temperature, at $800 \mathrm{~K}$, show such front transitions between an oxygen rich state of the surface and an oxygen poor state. The dark phase in these images can be associated with an oxygen covered surface area since adsorbed oxygen is known to strongly increase the work function $(\approx 1.1 \mathrm{eV}$ at maximum $) .{ }^{17}$ The bright phase reflecting a low work function could indicate a low adsorbate coverage or, alternatively, an area covered with ammonia fragmentation products $\mathrm{NH}_{\mathrm{x}}(\mathrm{x}=0-3)$. Ammonia on $\mathrm{Pt}$ is known to decrease the work function by more than $1 \mathrm{eV}$ below the level of the clean metal surface. ${ }^{6}$ Atomic nitrogen $(x=0)$ increases the work function by as much as $300 \mathrm{meV}$ at maximum but this value is still much lower than the $1.1 \mathrm{eV}$ reached with oxygen. ${ }^{17}$ Moreover, all kinds of $\mathrm{O}+\mathrm{NH}_{\mathrm{x}}(\mathrm{x}=0-3)$ coadsorbate phases have to be considered as well. Since the assignment of the bright phase is not clear, we speak in the following of an oxygen rich (dark area in PEEM) and of an oxygen poor (bright area in PEEM) phase.

The fronts in Fig. 1 are strongly elliptically distorted in the [110]-direction which is the direction in which the troughs of $\mathrm{Rh}(110)$ are oriented. The anisotropy in Fig. 1 is more or less what one would expect if the diffusional anisotropy just follows the geometric corrugation of the missing-row reconstructions. Diffusion should accordingly be fast along the $[1 \overline{1} 0]$-direction and slow in the direction perpendicular to the troughs which is the [001]-direction. If one initiates a transition in the reverse direction, i.e., from the dark phase to the bright phase in PEEM, one obtains the PEEM images displayed in Fig. 2. In order to reverse the front direction,

TABLE I. Overview of experiments conducted with PEEM and LEEM.

\begin{tabular}{lrrlr}
\hline \hline Temperature $(\mathrm{K})$ & $\mathrm{p}\left(\mathrm{O}_{2}\right)(\mathrm{mbar})$ & $\mathrm{p}\left(\mathrm{NH}_{3}\right)(\mathrm{mbar})$ & \multicolumn{1}{c}{ Observations } & Expt. technique \\
\hline 623 & $2.1-4.2 \times 10^{-6}$ & $6 \times 10^{-6}$ & Continuous transitions & LEEM \\
673 & $2.4 \times 10^{-6}$ & $5.1 \times 10^{-6}$ & Reaction fronts & LEEM \\
743 & $0.6 \times 10^{-6}$ & $4.6 \times 10^{-6}$ & Island patterns + secondary fronts & LEEM \\
740 & $1.35 \times 10^{-5}$ & $3.85 \times 10^{-5}$ & Traveling interface modulations & PEEM \\
800 & $2.7 \times 10^{-5}$ & $2.7 \times 10^{-5}$ & History dependent front & PEEM \\
& & & anisotropy & \\
\hline \hline
\end{tabular}




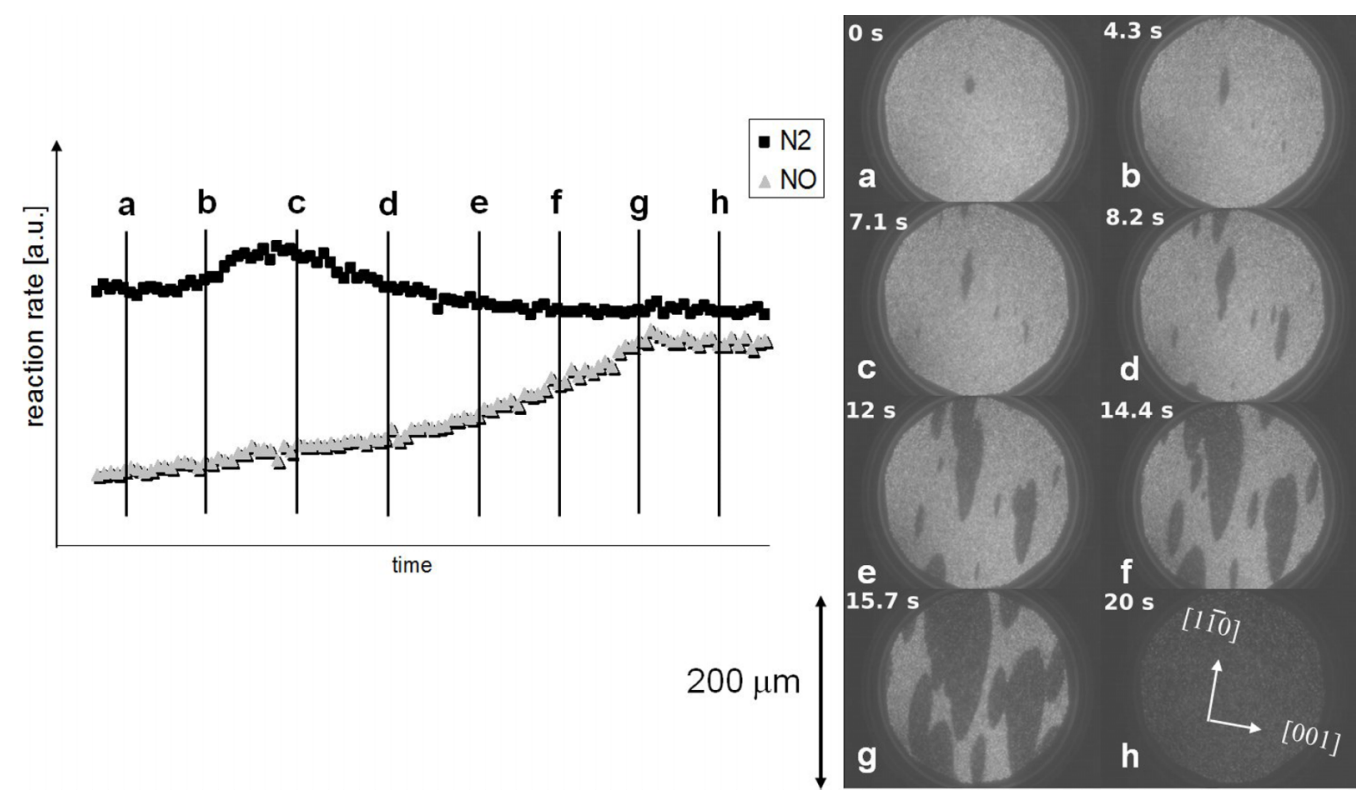

FIG. 1. Anisotropic front propagation during the transition from an oxygen poor state of the surface (bright) to an oxygen rich state (dark). The reaction front was initiated at $\mathrm{T}=800 \mathrm{~K}$ by raising $\mathrm{p}\left(\mathrm{O}_{2}\right)$ from $5 \times 10^{-6}$ to $2.7 \times 10^{-5} \mathrm{mbar}$ while $\mathrm{p}\left(\mathrm{NH}_{3}\right)$ was kept constant at $2.7 \times 10^{-5}$ mbar. The diagram on the left shows the subsequent evolution of the $\mathrm{N}_{2}$ and NO production rates and the panel on the right side shows PEEM images taken at the points marked in the rate diagram. The time interval between the first and the last frame ((a)-(h)) is $20 \mathrm{~s}$.
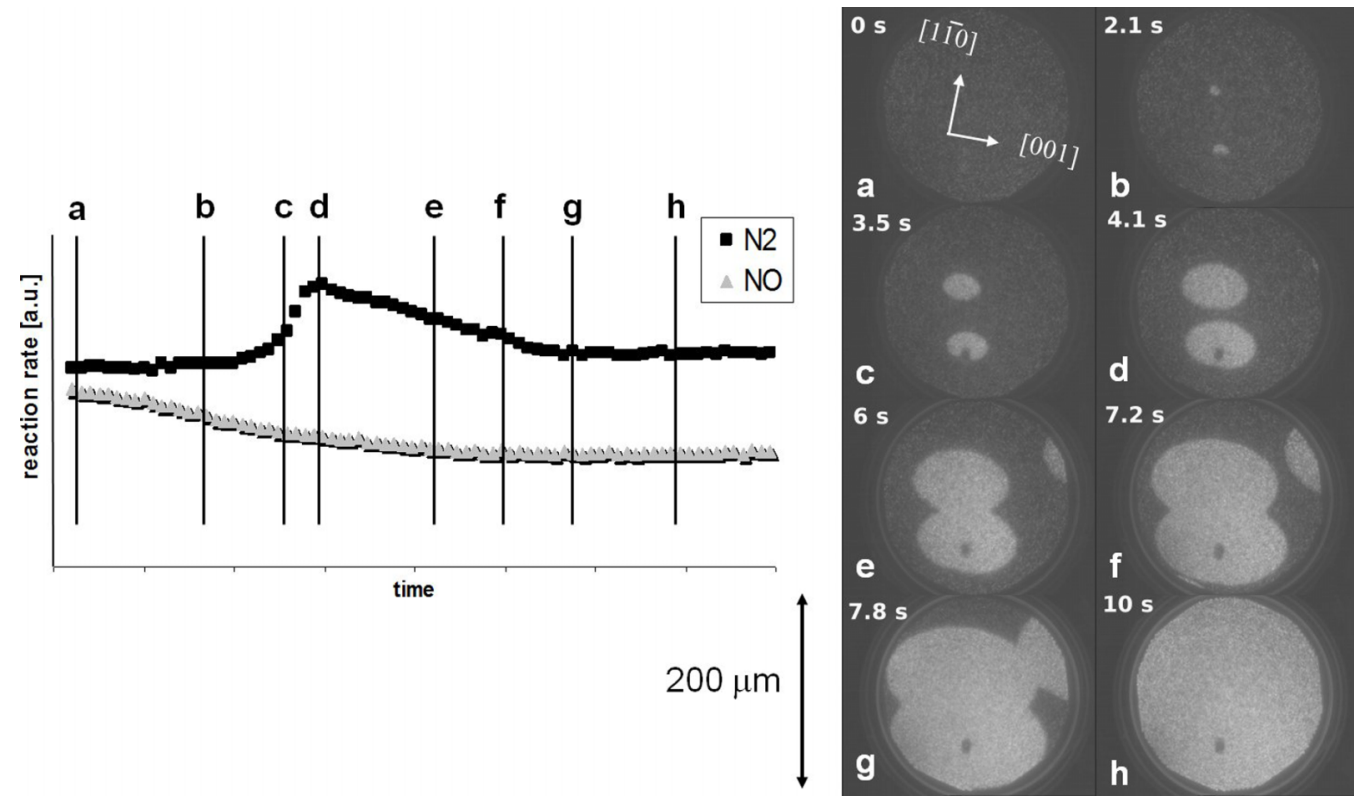

FIG. 2. Anisotropic front propagation during the transition from an oxygen rich state of the surface (dark) to an oxygen poor state (bright). The reaction front was initiated at $\mathrm{T}=800 \mathrm{~K}$ by decreasing $\mathrm{p}\left(\mathrm{O}_{2}\right)$ from $6 \times 10^{-5}$ mbar to $2.7 \times 10^{-5}$ mbar while $\mathrm{p}\left(\mathrm{NH}_{3}\right)$ was kept constant at $2.7 \times 10^{-5} \mathrm{mbar}$. The diagram on the left shows the evolution of the $\mathrm{N}_{2}$ and NO production rates and the panel on the right side shows PEEM images taken at the points marked in the rate diagram. The time interval between the first and the last frames ((a)-(h)) is $10 \mathrm{~s}$.

only a very small parameter change of the order of a few percent is required. The transitions in both directions are therefore located very close to the equistability point in the parameter space. Remarkably, despite nearly identical parameters, the anisotropy of the reaction front has been reversed and the long axis of the elliptical front is now oriented along the [001]-direction. For this change in the front anisotropy, no straightforward explanation exists since the small parameter change is unlikely to induce different types of reconstructions.
In order to assess whether the front anisotropy only depends on the direction of the transition as suggested by the results in Fig. 2, the following experiment is carried out. After initiating a reaction front like the one displayed in Fig. 1, the control parameter $\mathrm{p}\left(\mathrm{O}_{2}\right)$ is decreased halfway on completion of the transition white to black so that dark front does not expand further but starts to retract. The original anisotropy in that case does not change and the front retracts with the same shape as during expansion. So evidently, the anisotropy does not solely depend on the direction in which the front 
moves but the nucleation process already determines the front anisotropy.

The behavior of the $\mathrm{N}_{2}$ and NO production rates during front propagation is recorded in parallel to the PEEM measurements. The rate data in Figs. 1 and 2 show that the transition from the oxygen poor to the oxygen rich phase is associated with an increase in NO production and in the reverse direction with a decrease in the NO rate. Since it is the oxygen coverage which controls whether $\mathrm{N}_{2}$ or $\mathrm{NO}$ is formed, this correlation agrees with chemical intuition. In the $\mathrm{N}_{2}$ production, we observe in both directions a peak, i.e., a transient rise.

At low pressure, i.e., for $\mathrm{p}\left(\mathrm{NH}_{3}\right) \leq 1 \times 10^{-6} \mathrm{mbar}$, we also observe transitions between oxygen rich and oxygen poor but these transitions occur spatially homogeneously in PEEM, i.e., the PEEM intensity changes continuously. Supported also by the LEEM results (see Table I), one can conclude that low pressure/low temperature favors homogeneous transitions vs. transitions via reaction fronts. The term "homogeneous" here means homogeneous with respect to the resolution of the imaging technique. On a microscopic scale, the transitions are probably still inhomogeneous but with islands and reaction fronts below the spatial resolution of the microscope. The reason why macroscopic reaction fronts only develop at elevated temperature/pressure could lie in the reduced dynamics of the system at lower temperature/pressure.

\section{Travelling interface modulations}

At lower temperature, at $740 \mathrm{~K}$, the system is still globally bistable, but, in addition, we observe localized excitations at the interface of the oxygen rich/oxygen poor phase as shown in Fig. 3. ${ }^{9}$ For obtaining these excitations, one first has to adjust the oxygen partial pressure which serves as control parameter so that the interface does not shift its average position, i.e., the surface is at the point of equistability of the two phases. As demonstrated by the PEEM images in Fig. 3, one observes a number of localized excitations traveling along the interface with an average speed of $\approx 6 \mu \mathrm{m} / \mathrm{s}$ (see Video $\mathrm{S} 1$ in the supplementary material ${ }^{23}$ ). We find no connection between the crystallographic directions of the $\mathrm{Rh}(110)$ surface on one side and the orientation of the interface and the localized excitations on the other side. The localized excitations can be sustained for more than $1 \mathrm{~h}$.

The traveling interface modulations like the ones displayed in Fig. 3 are in general quite irregular. One might therefore suspect that fluctuations would be a more adequate description. However, these excitations which involve areas of about $100-400 \mu \mathrm{m}^{2}$ correspond to $10^{9}-10^{10}$ adparticles. This is a macroscopic quantity which is too large to be explained solely by thermal density fluctuations unless one devises a mechanism which amplifies these fluctuations so that they can reach a macroscopic size. Rather, we attribute the irregularity in the traveling interface modulations to structural imperfections of the surface which supposedly will have a particularly strong influence at the equistability point of two phases because the stability of the interface will be indifferent there with respect to a translational shift.

In the first report on the traveling interface modulations, it was suspected that the excitation mechanism is essentially the same as in the excitable $\mathrm{NO}+\mathrm{H}_{2}$ reaction on $\mathrm{Rh}(110){ }^{9}{ }^{9,18}$ This hypothesis was based on the assumption that directly at the interface, the same adsorbates should be present as in the $\mathrm{NO}+\mathrm{H}_{2}$ reaction, namely, atomic oxygen and atomic nitrogen. Due to the high desorption rate at $740 \mathrm{~K}$, the coverage of atomic hydrogen will be very small. The excitation cycle in the $\mathrm{NO}+\mathrm{H}_{2}$ reaction, $\mathrm{c}(2 \times 6)-\mathrm{O} \Rightarrow(3 \times 1) /(2 \times 1)-\mathrm{N}$ $\Rightarrow \mathrm{c}(2 \times 4)-2 \mathrm{O}, \mathrm{N} \Rightarrow \mathrm{c}(2 \times 6)-\mathrm{O}$, involves ordered adsorbate phases. ${ }^{17,18}$ An identification of these phases at the interface oxygen rich/oxygen poor in the $\mathrm{NH}_{3}+\mathrm{O}_{2}$ reaction would strongly support that this excitation cycle causes the interface modulations. With the conventional integral LEED system used in the PEEM-UHV system, in situ experiments were carried out. A $c(2 \times 6)$ and a $(1 \times 1)$ pattern were identified. However, the LEED experiments under reaction conditions could not be performed in the same pressure range of $10^{-5}$ mbar range where the localized excitations occurred, but only in the $10^{-6}$ mbar range. Moreover, since the probing area is about $1 \mathrm{~mm}^{2}$ which is very large compared to the extension of the interface (a few $\mu \mathrm{m}$ ), the results with a conventional

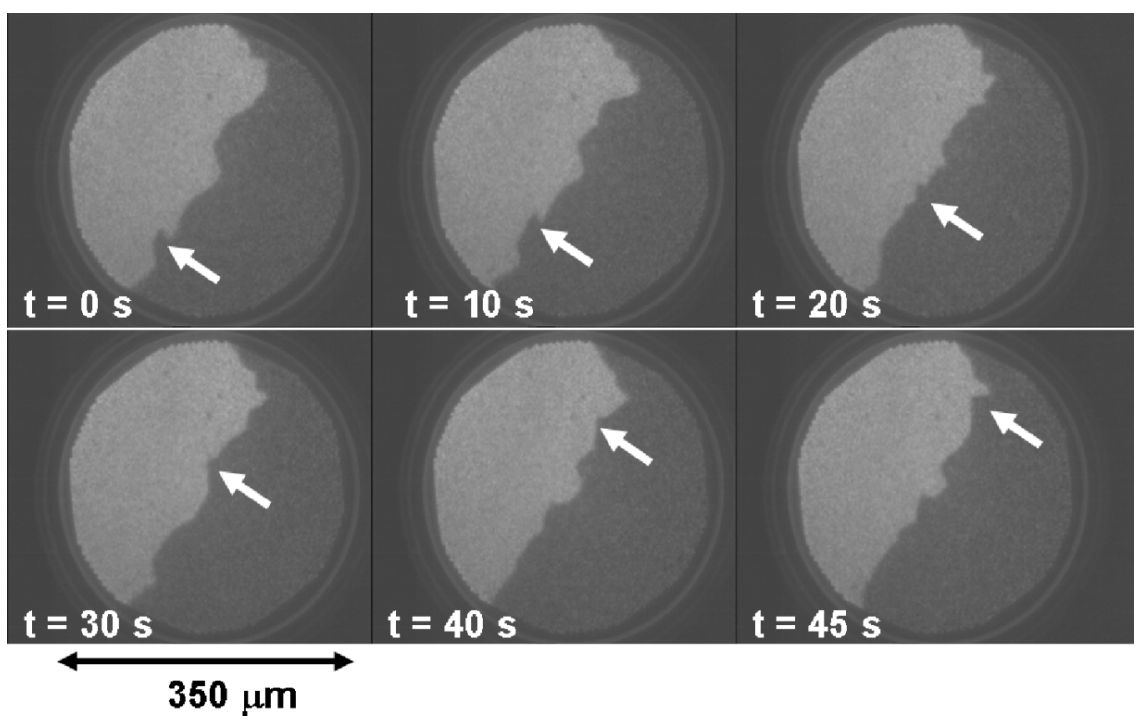

FIG. 3. PEEM images showing the propagation of interface excitations similar to a pulse under reaction conditions corresponding to the equistability point of the oxygen rich (dark) and oxygen poor phase (bright). The changing position of one such traveling pulse-like features has been marked with white arrows in the sequence. The crystallographic orientation of the surface is the same as in Figs. 1 and 2. Experimental conditions: $\mathrm{T}=740 \mathrm{~K}, \mathrm{p}\left(\mathrm{NH}_{3}\right)=3.85$ $\times 10^{-5}$ mbar, $\mathrm{p}\left(\mathrm{O}_{2}\right)=1.35 \times 10^{-5}$ mbar. 
LEED system are not really conclusive. Experiments with $\mu$-LEED which probes an area of only a few $\mu \mathrm{m}^{2}$ should therefore provide a definite answer.

\section{B. LEEM}

\section{Transitions without reaction fronts}

At relatively low temperature, at $620 \mathrm{~K}$, transitions between the oxygen rich and the oxygen poor phase occur without a macroscopic reaction front. The transitions proceed homogeneously in PEEM but the higher resolving LEEM reveals spatial structures as demonstrated by the images in Fig. 4. With $\mu$-LEED, the oxygen rich phase is identified as $\mathrm{c}(2 \times 6)-\mathrm{O}$ while the oxygen poor phase exhibits a $c(2 \times 4)$ pattern. This pattern is assigned to the mixed coadsorbate phase $c(2 \times 4)-\mathrm{O}, \mathrm{N}^{22}$ The only other known surface phase on $\operatorname{Rh}(110)$ with a $c(2 \times 4)$ pattern is a surface oxide, but since this surface oxide would require an oxygen concentration corresponding to several monolayers, this possibility is discarded here. ${ }^{24}$

Starting with the smooth surface of the $\mathrm{c}(2 \times 4)$ structure, a transition to the $\mathrm{c}(2 \times 6)-\mathrm{O}$ pattern is initiated by increasing $\mathrm{p}\left(\mathrm{O}_{2}\right)$ in the reaction mixture. The transition shown in Fig. 4 occurs in three consecutive steps. At the beginning, as shown in Fig. 4(a), only several stripes are faintly visible, indicated by arrows which represent steps or step bunches on the surface. Upon raising $\mathrm{p}\left(\mathrm{O}_{2}\right)$, the surface uniformly darkens. Then some small scale structures of the size of a few tenths of a $\mu \mathrm{m}$ appear as demonstrated by Fig. 4(b). The surface steps are still visible here. In the third stage, starting at many places simultaneously, these structures dissolve as shown by Fig. 4(c). Finally, a c $(2 \times 6)-\mathrm{O}$ structure results with a similar grainy surface as in Fig. 4(b) but with enhanced brightness (Fig. 4(d)). At this point, the original steps of the surface are no longer visible.

\section{Transitions with reaction fronts}

Increasing the sample temperature by $50 \mathrm{~K}$ from $620 \mathrm{~K}$ to $670 \mathrm{~K}$ changes the character of the transitions from a "homogeneous" transition without a macroscopic front to a transition with a macroscopic reaction front. As demonstrated by the LEEM images in Fig. 5, this transformation of the $\mathrm{c}(2 \times 6)$-O into the $\mathrm{c}(2 \times 4)$ via a front also involves three distinct stages. The LEEM images are taken in the dark field mode selecting a superstructure spot of the $c(2 \times 6)-O$ for imaging. Essentially, these stages appear to be the same as in the previously discussed transition without reaction front but in reverse order. By decreasing the oxygen partial pressure, first a transition from the grainy $\mathrm{c}(2 \times 6)-\mathrm{O}$ structure displayed in Fig. 5(a) to an apparently rougher surface shown in Fig. 5(b) is seen. Next, the surface considerably smoothens but it is in this step that a reaction front appears. In Fig. 5(c), this front which separates the still roughened part of the surface from the smoothened area travels from right to left. Its position is marked by two arrows. In the final transition, a $c(2 \times 4)$ structure is formed. Due to dark field contrast, the $\mathrm{c}(2 \times 4)$ structure is imaged as dark area on the surface. This last transition occurs also via a traveling reaction front moving from right to left in Fig. 5(d). By carefully adjusting the
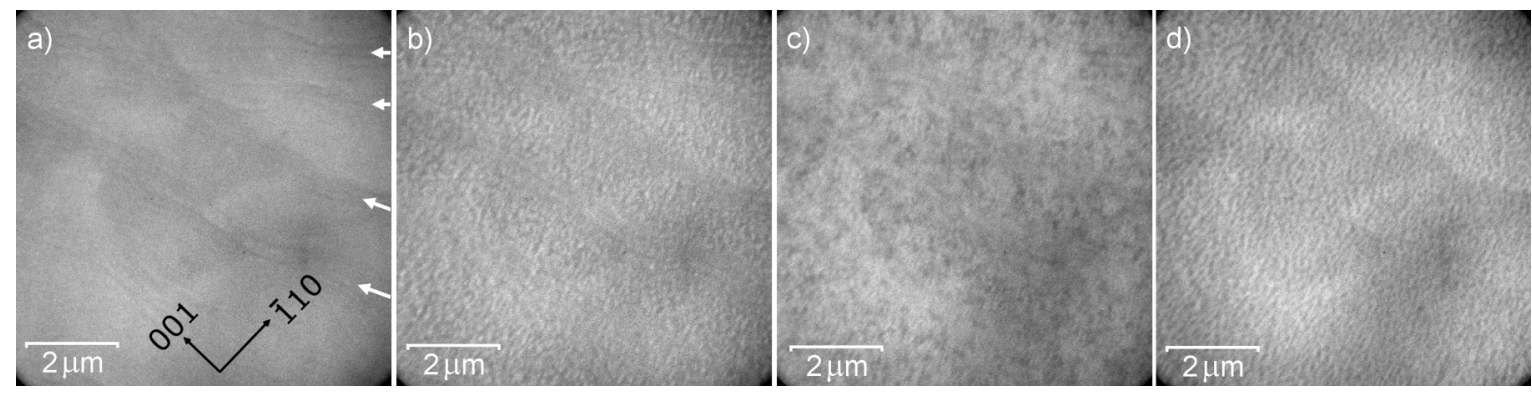

FIG. 4. LEEM images showing a transition without reaction front from a surface with a c $(2 \times 4)$ pattern to the $\mathrm{c}(2 \times 6)$-O structure of adsorbed oxygen. The transition is initiated at $\mathrm{T}=620 \mathrm{~K}$ by raising $\mathrm{p}\left(\mathrm{O}_{2}\right)$ stepwise from $0.2 \times 10^{-6}$ mbar in (a) to $4.6 \times 10^{-6} \mathrm{mbar}$ in $(\mathrm{d})$ while $\mathrm{p}\left(\mathrm{NH}_{3}\right)$ is kept constant at $6 \times 10^{-6} \mathrm{mbar}$. $\mathrm{p}\left(\mathrm{O}_{2}\right)$ in (b) and (c) is $1.6 \times 10^{-6}$ mbar and $3.6 \times 10^{-6}$ mbar, respectively. The white arrows in (a) indicate step bunches. The primary electron energy was 10.7 eV.
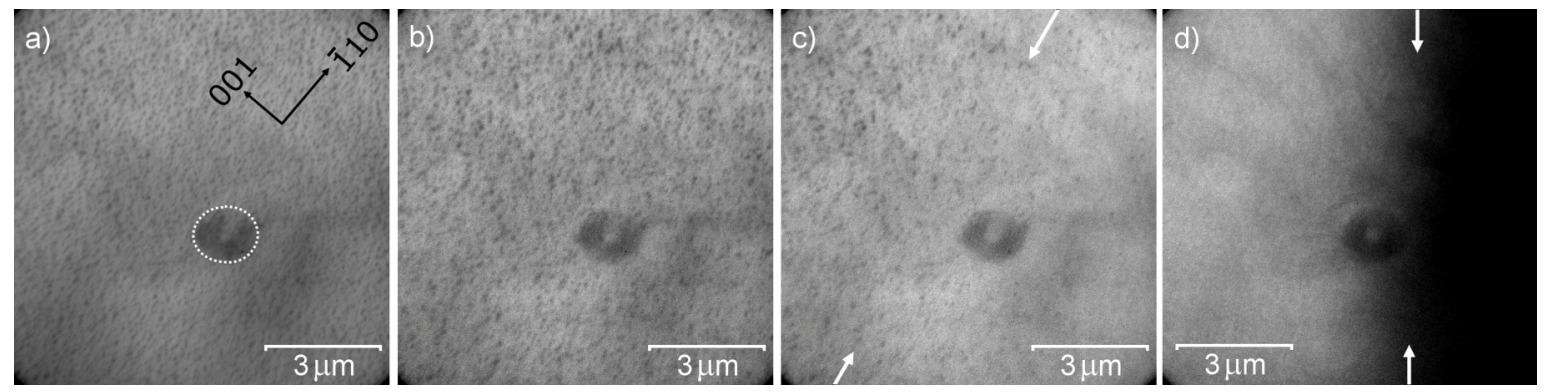

FIG. 5. Dark-field LEEM images showing the front-mediated transition from the $\mathrm{c}(2 \times 6)-\mathrm{O}$ surface to a surface with $\mathrm{c}(2 \times 4)$ pattern at $\mathrm{T}=670 \mathrm{~K}$. The transition is initiated by slowly decreasing $\mathrm{p}\left(\mathrm{O}_{2}\right)$ from $2.7 \times 10^{-6}$ mbar while the ammonia partial pressure is fixed at $\mathrm{p}\left(\mathrm{NH}_{3}\right)=5.1 \times 10^{-6}$ mbar. The dotted circle in (a) marks a surface defect. The position of the two consecutive reaction fronts are marked with arrows in (c) and (d), respectively. A superstructure beam of the $\mathrm{c}(2 \times 6)-\mathrm{O}$ structure is used for imaging. The primary electron energy is $14.9 \mathrm{eV}$. 
oxygen partial pressure, such reaction fronts can be slowed down and even brought to a complete stop. Such frozen configurations of coexisting phases are used to identify the $c(2 \times 6)-O$ and $c(2 \times 4)$ structures, respectively, on either sides of the front with $\mu$ LEED.

In the first step of the transformation process, the reaction of adsorbed oxygen with ammonia apparently creates some defects and holes in the $\mathrm{c}(2 \times 6)-\mathrm{O}$ structure appearing as roughness in the LEEM images. Whether the apparent roughness is due to a real roughening or just a change in reflectivity is difficult to tell from the LEEM images. Since a change in oxygen coverage leads to a different type of reconstruction requiring a mass transport of $\mathrm{Rh}$ atoms to occur, a real roughening would be plausible. ${ }^{21}$ The long range order of the oxygen structure is only destroyed in a subsequent step when a reaction front transforms the surface into a structure which appears black in dark field imaging. This front appearing as interface grey/dark area in Fig. 5(d) marks the structural transition from the $\mathrm{c}(2 \times 6)-\mathrm{O}$ to a $\mathrm{c}(2 \times 4)$. It is a reduction front associated with a decrease of the oxygen coverage. This reduction front, however, is a secondary front. The primary front is the front visible in Fig. 5(c) which runs ahead of the reduction front and is associated with an apparent smoothing of the surface. The nature of this front is not clear but, tentatively, one could associate the front with a partial reduction of the oxygen covered surface that preserves the $c(2 \times 6)$ long range order.

\section{Island formation}

At elevated temperature, at $740 \mathrm{~K}$ in the $10^{-6}$ mbar range, an inhomogeneous state of the surface results under reaction conditions. Remarkably, in this state, despite a large excess of ammonia in the gas phase, mesoscopic oxygen islands with a $\mathrm{c}(2 \times 6)-\mathrm{O}$ structure persist on the surface. Starting from a homogeneous reduced state of the surface, the preparation procedure involves first increasing the oxygen partial pressure in the reaction mixture well beyond a limit where oxygen islands form on the $\mathrm{Rh}(110)$ surface. A subsequent strong reduction of $\mathrm{p}\left(\mathrm{O}_{2}\right)$ to only $3 \%$ of its maximum value does not remove the oxygen islands. While they survive each island shrinks as shown in Fig. 6, thus reducing the fraction of the surface area covered by these islands from $75 \%$ to 25\%. The LEEM image in Fig. 6(c) shows a dark area still densely populated with bright islands of a diameter in the submicrometer range. $\mu$-LEED demonstrates that the bright islands are in fact oxygen islands with a $\mathrm{c}(2 \times 6)-\mathrm{O}$ structure.

A closer inspection of the islands in Fig. 6 reveals that nearly all of them contain one or several dark spots in the interior. Upon further reducing $\mathrm{p}\left(\mathrm{O}_{2}\right)$, a macroscopic reaction front is ignited which is displayed in Fig. 7. The course of the front can be followed by the bright islands which are converted by the front into dark islands. In the area surrounding the islands, the front hardly causes an intensity change but this is due to the specific imaging conditions selected here; in fact, the front also transforms the surrounding area. When the macroscopic front reaches a hitherto unconverted island, a subordinate reaction front is ignited on that island, transforming the bright island into a dark one. In most cases, ignition occurs at the boundary of the island, but sometimes also in the interior. These secondary reaction fronts inside the islands are strongly influenced by surface topography such as surface steps. Moreover, the secondary fronts do not necessarily travel in the same direction as the macroscopic primary front. The overall result is that oxygen islands are completely transformed into the oxygen poor phase, the $c(2 \times 4)$, i.e., the reaction fronts are reduction fronts.

LEEM images with higher magnification in Fig. 8 show in detail how the progression of the macroscopic front initiates the conversion of the oxygen islands via secondary fronts (see Video S2 in the supplementary material ${ }^{23}$ ). As seen before in Figs. 6 and 7, the island transformation starts by island shrinking (Fig. 8(a)), followed by secondary reaction front nucleating inside each island (Fig. 8(b)) and growing until only tiny fragments of the former oxygen islands remain (Fig. 8(d)). The overview in Fig. 8(e) is recorded shortly after Fig. 8(b) and shows the macroscopic primary front approaching the islands marked with a white square. As demonstrated by Fig. 8(d), even after the reaction front has passed, the shape of the islands remains still visible as a shadow, indicating a memory effect.

Selecting a different energy where oxygen islands are imaged as dark areas and the surrounding area as bright, one obtains the series reproduced in Fig. 9. First, the $\mathrm{c}(2 \times 6)$
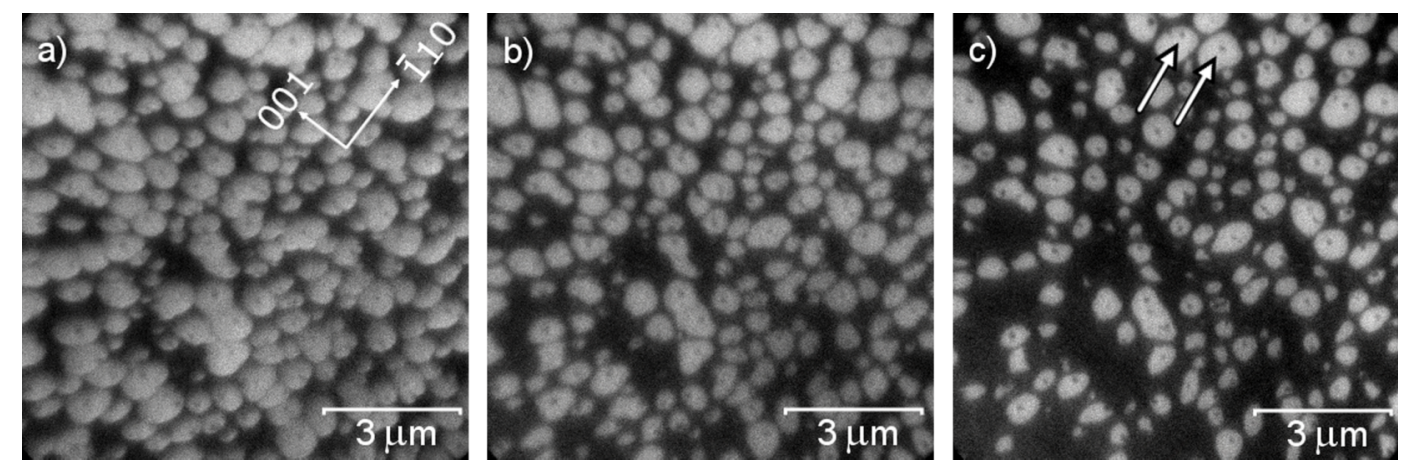

FIG. 6. Dark-field LEEM images showing shrinking oxygen islands at $\mathrm{T}=740 \mathrm{~K}$, caused by a drastic reduction of the oxygen partial pressure from $\mathrm{p}\left(\mathrm{O}_{2}\right)=2.1 \times 10^{-5}$ mbar to $\mathrm{p}\left(\mathrm{O}_{2}\right)=0.7 \times 10^{-6}$ mbar. $\mathrm{p}\left(\mathrm{NH}_{3}\right)$ is kept constant at $4.6 \times 10^{-6}$ mbar. Further reduction of oxygen partial pressure initiates reaction fronts shown below in Figs. 7 and 8 . The arrows in (c) mark two dark spots on oxygen islands. A beam of the c(2 $\times 6)-\mathrm{O}$ structure is used for imaging. The primary electron energy is $3.7 \mathrm{eV}$. 

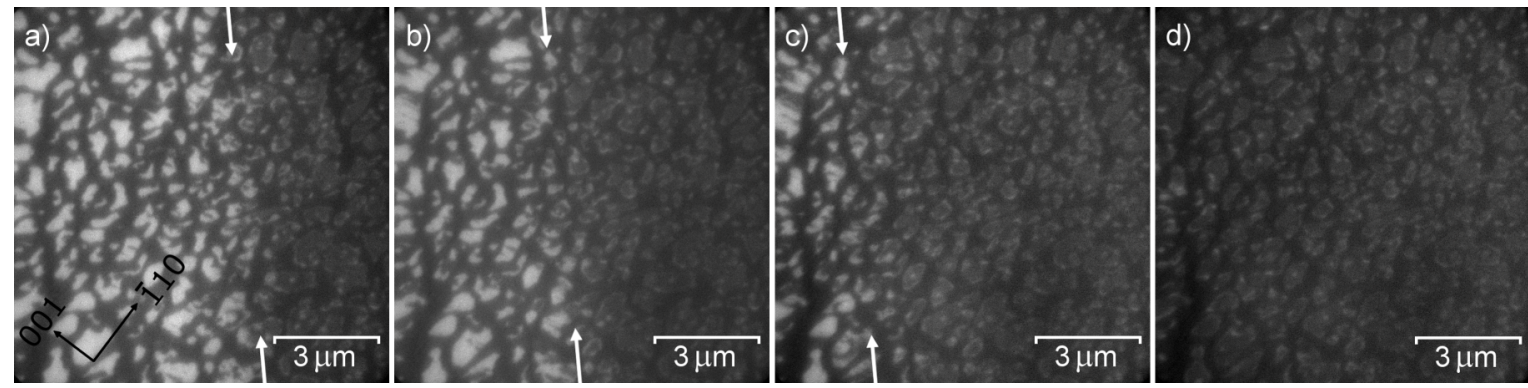

FIG. 7. Macroscopic reaction front passing over oxygen islands at $\mathrm{T}=740 \mathrm{~K}$. After preparation of the inhomogeneous surface displayed in Fig. 6, a macroscopic reaction front is initiated by a further decrease of $\mathrm{p}\left(\mathrm{O}_{2}\right)$ below $1 \times 10^{-6} \mathrm{mbar}$. The position of the front is indicated by arrows. The speed of the macroscopic front is $\sim 75 \mathrm{~nm} / \mathrm{s}$. $\mathrm{p}\left(\mathrm{NH}_{3}\right)$ and imaging conditions as in Fig. 6 except that the primary electron energy is set to $4.6 \mathrm{eV}$.
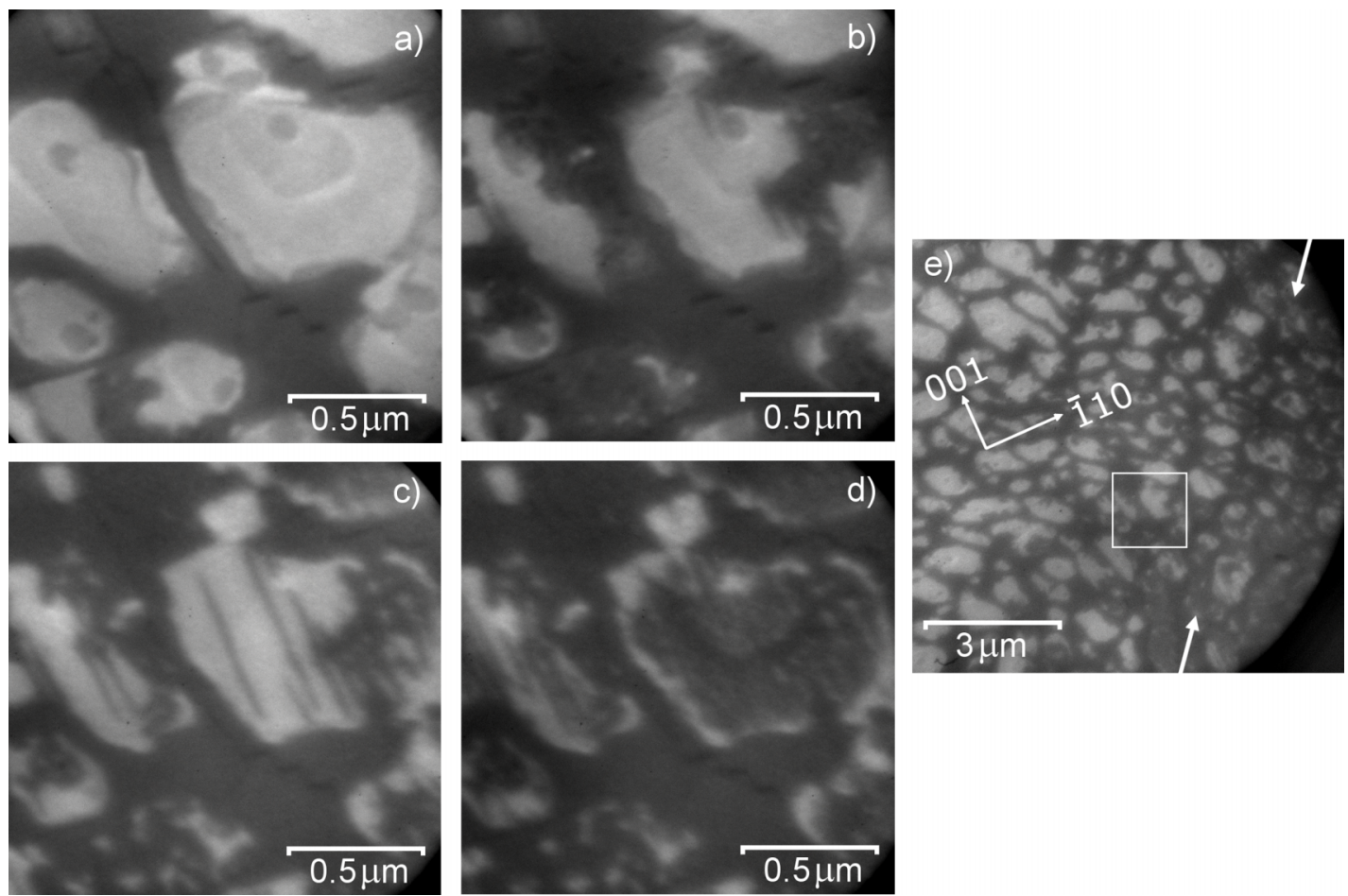

FIG. 8. Detailed view of the island transformation as displayed in Figs. 6 and 7 showing how the microscopic fronts progress on the oxygen islands. The position of the macroscopic reaction front is indicated by arrows in the overview in (e), which is recorded between (b) and (c). The square marks the area seen in (a)-(d) (see text). Experimental conditions are the same as in Fig. $6\left(\mathrm{~T}=740 \mathrm{~K}, \mathrm{p}\left(\mathrm{NH}_{3}\right)=4.6 \times 10^{-6} \mathrm{mbar}, \mathrm{p}\left(\mathrm{O}_{2}\right)<1 \times 10^{-6}\right.$ mbar $)$ except that the primary electron energy is set to $10 \mathrm{eV}$.
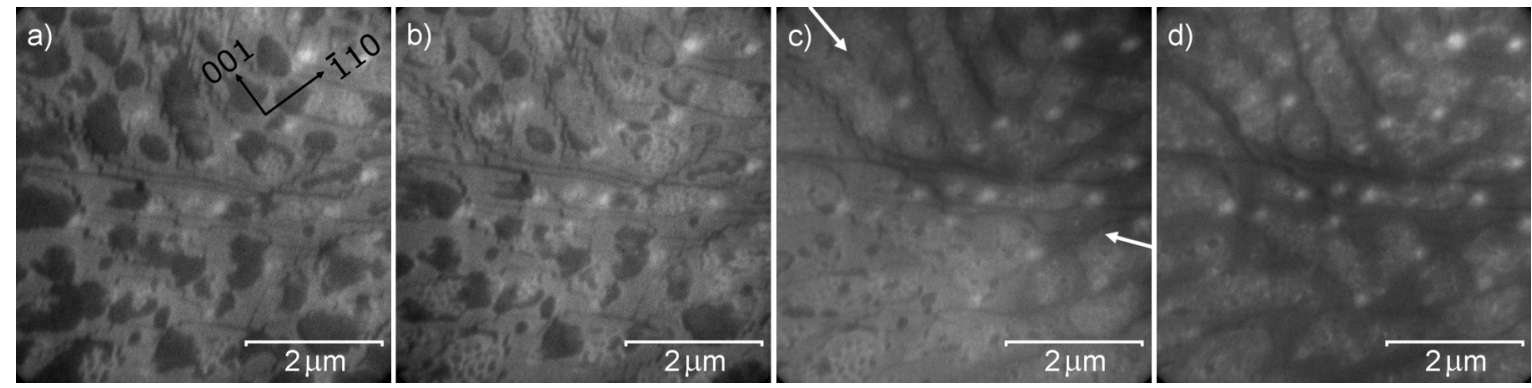

FIG. 9. Primary and secondary reaction fronts transforming oxygen islands and surrounding area. The process and the experimental conditions are the same as in Figs. 6 and 7 but a different electron energy of $7.4 \mathrm{eV}$ is chosen which leads to better contrast in the area surrounding the oxygen islands. After conversion of the oxygen islands (b), a secondary reaction front indicated by arrows in (c) flows around the positions of former islands creating a kind of network structure (d).

islands visible in Figs. 9(a) and 9(b) are converted. In a second step, shown in Figs. 9(c) and 9(d), a reaction front propagating through the imaged area from right to left transforms the channel network surrounding the original $\mathrm{c}(2 \times 6)$ islands to a darker grey level. Furthermore, one observes a number of bright circular spots distributed irregularly over the imaged surface. Their size and position do not visibly change during the transformation process. In the last image, they appear 
more prominent but this is only an apparent change due to the enhanced contrast with the surrounding dark area. Inside the channel network, one realizes dark lines going straight or sometimes going zig-zag. These lines represent atomic steps or step bunches of the surface. The bright spots might be related to certain topographical features like hillocks exhibiting an enhanced brightness.

The memory effect visible in the bright field images of the $(2 \times 6)-\mathrm{O}$ indicates that after the reactive removal of oxygen, either a structural or a chemical modification of the surface took place. A chemical modification by either oxide or subsurface oxygen formation cannot be excluded, but since no further evidence for such a chemical modification exists at present, a structural modification appears to be more likely. A different amount of structural disorder on the former $c(2 \times 6)-O$ islands and the surrounding area could lead to a different reflectivity, thus explaining the memory effect.

\section{DISCUSSION}

\section{A. Identification of the adsorbate phases and mechanism of traveling interface modulations}

The excitation mechanism for pulses in the system $\mathrm{Rh}(110) / \mathrm{NO}+\mathrm{H}_{2}$ involves a cyclic transformation $\mathrm{c}(2 \times 6)-\mathrm{O}$ $\Rightarrow(3 \times 1) /(2 \times 1)-\mathrm{N} \Rightarrow \mathrm{c}(2 \times 4)-2 \mathrm{O}, \mathrm{N} \Rightarrow \mathrm{c}(2 \times 6)-\mathrm{O}$ where the pure oxygen phase is the resting state, the pure nitrogen phases the excited state, and the N,O-coadsorption phase the refractory state. ${ }^{17,18}$ The original expectation had been that only at the interface oxygen poor/oxygen rich phase, all three components of the excitation cycle are present, thus explaining the localized excitability. ${ }^{9}$ So far, only the $c(2 \times 4)$ and the $c(2 \times 6)$-O have been identified under reaction conditions high enough in temperature. Since, however, no in situ LEED/ $\mu$-LEED experiments were conducted under conditions under which traveling interface modulations were observed, a possible participation of a pure nitrogen phase cannot be definitely excluded. The above cited mechanism can therefore not be completely ruled out.

The existence of a $\mathrm{c}(2 \times 4)-2 \mathrm{O}, \mathrm{N}$ phase does not really fit into what is known about the stability of different nitrogen phases on $\mathrm{Rh}(110)$. In $\mathrm{N}_{2}$ thermal desorption spectra, the peak maximum of the $\mathrm{c}(2 \times 4)-2 \mathrm{O}, \mathrm{N}$ coadsorption phase lies at $530 \mathrm{~K}$ which is more than $90 \mathrm{~K}$ lower than the peak maximum of the pure nitrogen phase $(2 \times 1)-\mathrm{N}$ at $620 \mathrm{~K}^{17,25}$ The presence of repulsive interactions between coadsorbed oxygen and nitrogen explains well the destabilization of nitrogen in the coadsorbed phase. Surprisingly, we see in ammonia oxidation the $\mathrm{c}(2 \times 4)$ coadsorbate phase and not the more stable pure nitrogen phases. Two possible explanations can be offered. Either the $\mathrm{c}(2 \times 4)$ we observe is not the $\mathrm{c}(2 \times 4)-2 \mathrm{O}, \mathrm{N}$ described in the literature but it is due to some unknown adsorbate phase or the oxygen in the $\mathrm{c}(2 \times 4)-2 \mathrm{O}, \mathrm{N}$ has besides repulsive interactions also a stabilizing effect on the nitrogen in the coadsorbate phase. Kinetically, for example, the oxygen can stabilize nitrogen by facilitating $\mathrm{NH}_{3}$ decomposition. Since oxygen on Pt has been shown to activate ammonia decomposition such an effect could also exist for Rh. ${ }^{11,13}$
The traveling interface modulations (see Fig. 3) have been simulated in a general mathematical 3-variable model in which the excitability of a subsystem is coupled to the position of the interface. ${ }^{9}$ For formulating a realistic mathematical model, first the mechanism for the interface modulations needs to be established. The expectation to find an excitation mechanism quite analogous to $\mathrm{NO}+\mathrm{H}_{2}$ on $\mathrm{Rh}(110)$ has so far not been supported by experiments because the pure nitrogen phases $(3 \times 1) /(2 \times 1)-\mathrm{N}$ have not been detected under the reaction conditions of travelling interface modulations. The possibility still exists that in situ measurements at higher pressure, in the $10^{-5}$ or $10^{-4}$ mbar range, may detect the nitrogen phase missing for a confirmation of an excitation cycle analogous to the $\mathrm{NO}+\mathrm{H}_{2}$ reaction on $\mathrm{Rh}(110)$. Alternatively, one can consider another possibility. A unique feature of the present system is the traveling interface modulations, and another unique feature is the history dependent change in front anisotropy. This coincidence suggests that both phenomena could have a common mechanistic origin. A strategy to establish the mechanism for the travelling interface modulations would therefore be to first try to solve the problem of the changing front anisotropy.

\section{B. History dependent front anisotropy}

The front velocity, $c_{f}$, of a reaction front in a reactiondiffusion system can be written as $c_{f} \sim \sqrt{ }\left(D_{x} K_{e f f}\right)$, where $D_{x}$ is the diffusion constant of the velocity limiting species and $\mathrm{K}_{\text {eff }}$ is an effective rate constant summarizing the various reaction steps involved. ${ }^{26}$ On an anisotropic surface, the diffusion anisotropy in $\mathrm{D}_{\mathrm{x}}$ will lead to an elliptically shaped reaction front. If several species with different anisotropies are present or when energetic interactions or reconstructions of the substrate make the diffusional anisotropy state-dependent, more complicated effects can arise because a simple rescaling of the coordinate axes will not remove the diffusional anisotropy. For example, rectangularly shaped patterns may form in such a case. ${ }^{16}$ In ammonia oxidation over $\mathrm{Rh}(110)$, we observe a change in the anisotropy depending on whether the oxygen rich phase nucleates and expands into the oxygen poor phase or whether the opposite process takes place. In a system with simple anisotropy, i.e., in systems with one velocity-limiting diffusing species and constant diffusional anisotropy, such an effect would not be observed. Both fronts would exhibit the same anisotropy.

A similar behavior as in ammonia oxidation has been found in the $\mathrm{O}_{2}+\mathrm{H}_{2}$ reaction over $\mathrm{Rh}(110) .^{27,28}$ There, reaction fronts initiating the transition from oxygen-covered to oxygen-free surfaces were elliptically elongated in the [1 10$]$ direction at lower temperature and elliptically elongated in the [001]-direction at elevated temperatures. The change in the anisotropy was explained with hydrogen diffusion being ratelimiting at lower temperature and oxygen diffusion becoming rate-limiting at higher temperature. There are in fact two decisive differences between the anisotropy changes observed in ammonia oxidation and the $\mathrm{O}_{2}+\mathrm{H}_{2}$ reaction over $\mathrm{Rh}(110)$. First, in the $\mathrm{O}_{2}+\mathrm{H}_{2}$ reaction, the switching anisotropy was always observed for reduction fronts whereas in ammonia oxidation, the different anisotropies were seen for a reduction 
and an oxidation front. Second, in the $\mathrm{O}_{2}+\mathrm{H}_{2}$ reaction, the change in anisotropy was caused by a large change of the parameter range whereas in ammonia oxidation due the very narrow bistability range, the parameter change was very small. The small change of the control parameter can hardly account for the drastic change in anisotropy.

Another quite obvious difference between the two systems is the additional presence of nitrogen in the form of $\mathrm{NH}_{\mathrm{x}}(\mathrm{x}=0-3)$ species in the $\mathrm{NH}_{3}+\mathrm{O}_{2}$ reaction. How the nitrogen affects the front anisotropy is not clear. The pure nitrogen phases have so far not been identified under reaction conditions and the reconstruction of the mixed c $(2 \times 4)-2 \mathrm{O}, \mathrm{N}$ coadsorbate phase is of the same type as of the pure oxygen phases, namely, of the missing row type. Adsorbate-adsorbate interactions between $\mathrm{O}$ and $\mathrm{N}$ atoms might influence the diffusional anisotropy but other than that they are repulsive no detailed information is available.

In addition, we have to take into account structural intermediates in the reaction of oxygen with ammonia discovered in a scanning tunneling microscopy (STM) study where the titration of the oxygen covered $\mathrm{Rh}(110)$ surface with ammonia was followed at room temperature and after annealing to $450 \mathrm{~K}^{12}$ It was observed that the transformation of the $\mathrm{c}(2 \times 8)-\mathrm{O}\left(\theta_{\mathrm{O}}=0.75\right)$ to the $(2 \times 1)-\mathrm{N}$ proceeds via an intermediate with a local $\mathrm{c}(2 \times 6)$ symmetry. This intermediate consists of Rh-N trimers oriented in the [001]direction. If the intermediate is short-living, it will not show up in LEED, but it is well conceivable that the structural intermediates influence the diffusional anisotropy. Another possible influence on diffusional anisotropy is the formation of atomic steps due to the necessary mass transport of $\mathrm{Rh}$ atoms as the surface undergoes a transformation into a different type of reconstruction.

At present, it is not clear which of the different possible factors is actually responsible for the history dependent change in front anisotropy. Further, in situ experiments with $\mu$ LEED establishing which structures are actually associated with the change in front anisotropy are required in order to obtain a firm basis for formulating a mechanism.

\section{Island formation and secondary reaction fronts}

A rather surprising observation is that even with a large excess of ammonia, oxygen islands still persist on the surface, as demonstrated by the LEEM images in Figs. 6-9. Stationary concentration patterns can arise in a reactiondiffusion systems due to a Turing instability or, if energetic interactions dominate, they may develop as a consequence of reactive phase separation. ${ }^{26,29}$ Since the islands do not look like a regular pattern and since basic requirements like the existence of an intrinsic wavelength have not been established, it is probably of little use to try to classify the islands as a certain type of non-equilibrium structure. Moreover, these mechanisms would require the islands to evolve smoothly out of the homogeneous state of the surface which does not agree with the experimental observations. Instead, a simple possible feedback mechanism is considered which can stabilize oxygen islands. If we assume that the probability for dissociative chemisorption of ammonia is higher over an oxygen adlayer with defects than over a perfectly ordered oxygen adlayer, then regions of high structural perfection will be stabilized. This will occur because a positive feedback exists between adsorption of ammonia in regions with defects and further growth of the number of defects due to reaction. In the end, oxygen islands with a high structural perfection will be surrounded by areas with low oxygen coverage. This picture is in qualitative agreement with the LEEM images shown in Figs. 6-9.

In the LEEM images of Figs. 6-9, one observes a primary front transforming the area surrounding the islands and secondary fronts inside the islands. Both fronts are reduction fronts but the primary front can evidently propagate faster than the secondary fronts inside the islands. A possible reason is that the area outside the islands might already be partially depleted of oxygen. Reaction fronts inside the high oxygen coverage islands are more difficult to nucleate due to a higher oxygen coverage and only when the primary front touches the edge of such an oxygen island, a secondary front inside the island is ignited. What is missing in this mechanistic picture is a precise identification of the reducing species which could be $\mathrm{H}_{\mathrm{ad}}$ formed by dissociating ammonia but also a mobile $\mathrm{NH}_{\mathrm{x}}$ species $(\mathrm{x}=1-3)$.

\section{CONCLUSIONS}

Front propagation in the bistable $\mathrm{NH}_{3}+\mathrm{O}_{2}$ reaction over $\mathrm{Rh}(110)$ has been investigated with PEEM and LEEM as spatially resolving methods. One unique feature of this bistable system is that the anisotropy of front propagation is history dependent, i.e., it varies depending on the direction of the transition. A second unique feature is that we observe localized excitations traveling along the interface of two surface phases. With $\mu$-LEED, a c $(2 \times 6)-O$ structure and a $\mathrm{c}(2 \times 4)$ pattern have been identified but not the pure nitrogen phases which would be required in order to construct an excitation cycle analogous to the $\mathrm{NO}+\mathrm{H}_{2}$ reaction on $\mathrm{Rh}(110)$. Nevertheless, the coincidence of the traveling interface modulations and of the history dependent front anisotropy suggests that both phenomena have a common mechanistic origin. The LEEM results have demonstrated that below the macroscopic level of a reaction front, a microscopic substructure can exist involving island formation and secondary reaction fronts. Even with a large excess of ammonia, oxygen islands can persist in a reacting atmosphere as stable structures. By a further parameter change, one can then initiate macroscopic (primary) reduction fronts transforming the surroundings of the islands and microscopic (secondary) reduction fronts converting the interior of the oxygen islands. Memory effects and changes in the topographical features suggest that roughening by reaction-induced mass transport of $\mathrm{Rh}$ atoms plays a significant role, but in order to substantiate this claim further studies are required.

\section{ACKNOWLEDGMENTS}

M.R. gratefully acknowledges CONICET-UNLP (Argentina) and DFG-DAAD (Germany) for providing 
scholarships and financial assistance (Project No. D768-2015

"Cooperación Internacional CONICET/DFG-Q").

${ }^{1}$ T. H. Chilton, The Manufacture of Nitric Acid by the Oxidation of Ammonia, Chemical Engineering Progress Monograph Series Vol. 3 (American Institute of Chemical Engineers, New York, 1960).

${ }^{2}$ E. S. J. Lox and B. H. Engler, in Handbook of Heterogeneous Catalysis, edited by G. Ertl, H. Knözinger, and J. Weitkamp (VCH, Weinheim, 1997), Vol. 4.

${ }^{3}$ R. Imbihl and G. Ertl, Chem. Rev. 95, 697 (1995).

${ }^{4}$ R. Imbihl, in Handbook of Surface Science, edited by E. Hasselbrink and B. Lundquist (Elsevier, Amsterdam, 2008), Vol. 3, Chap. 9, pp. 341-428.

${ }^{5}$ T. Katona and G. A. Somorjai, J. Chem. Phys. 96, 5465 (1992).

${ }^{6}$ M. Rafti, F. Lovis, Y. Zeng, and R. Imbihl, Chem. Phys. Lett. 446, 323 (2007).

${ }^{7}$ M. L. Wagner and L. D. Schmidt, J. Phys. Chem. 99, 805 (1995).

${ }^{8}$ M. Rafti, F. Lovis, and R. Imbihl, Catal. Lett. 142, 16 (2012).

${ }^{9}$ M. Rafti, H. Uecker, F. Lovis, V. Krupennikova, and R. Imbihl, Phys. Chem. Chem. Phys. 14, 5260 (2012).

${ }^{10}$ J. M. Bradley, A. Hopkinson, and D. A. King, J. Phys. Chem. 99, 17032 (1995).

${ }^{11}$ R. Imbihl, A. Scheibe, Y. F. Zeng, S. Gunther, R. Kraehnert, V. A. Kondratenko, M. Baerns, W. K. Offermans, A. P. J. Jansen, and R. A. Van Santen, Phys. Chem. Chem. Phys. 9, 3522 (2007).

${ }^{12}$ M. Kiskinova, A. Baraldi, R. Rosei, V. R. Dhanak, G. Thornton, F. Leibsle, and M. Bowker, Phys. Rev. B 52, 1532 (1995).
${ }^{13}$ W. K. Offermans, A. P. J. Jansen, and R. A. v. Santen, Surf. Sci. 600, 1714 (2006).

${ }^{14}$ D. G. Löffler and L. D. Schmidt, J. Catal. 41, 440 (1976); Surf. Sci. 59, 195 (1976).

${ }^{15}$ R. Kraehnert and M. Baerns, Chem. -Eng. J. 137, 361 (2008); J. Pérez Ramírez and E. V. Kondratenko, Catal. Today 121, 160 (2007).

${ }^{16} \mathrm{~F}$. Mertens and R. Imbihl, Nature 370, 124 (1994).

${ }^{17}$ F. Mertens, S. Schwegmann, and R. Imbihl, J. Chem. Phys. 106, 4319 (1997).

${ }^{18}$ A. Makeev, M. Hinz, and R. Imbihl, J. Chem. Phys. 114, 9083 (2001).

${ }^{19}$ H. H. Rotermund, Surf. Sci. Rep. 29, 265 (1997).

${ }^{20}$ Th. Schmidt, S. Heun, J. Slezak, J. Diaz, K. C. Prince, G. Lilienkamp, and E. Bauer, Surf. Rev. Lett. 5, 1287 (1998).

${ }^{21}$ G. Comelli, V. R. Dhanak, M. Kiskinova, K. C. Prince, and R. Rosei, Surf. Sci. Rep. 32, 165 (1998)

${ }^{22}$ M. Gierer, F. Mertens, H. Over, G. Ertl, and R. Imbihl, Surf. Sci. 339, L903 (1995).

${ }^{23}$ See supplementary material at http://dx.doi.org/10.1063/1.4935274 for videos of traveling interface modulations and of secondary reaction fronts transforming oxygen islands.

${ }^{24}$ C. Dri, C. Africh, F. Esch, G. Comelli, O. Dubay, L. Köhler, F. Mittendorfer, G. Kresse, P. Dudin, and M. Kiskinova, J. Phys. Chem. 125, 94701 (2006).

${ }^{25}$ G. Comelli, S. Lizzit, P. Hofmann, G. Paolucci, M. Kiskinova, and R. Rosei, Surf. Sci. 277, 31 (1992).

${ }^{26}$ A. S. Mikhailov, Foundations of Synergetics I (Springer, Berlin, 1994).

${ }^{27}$ F. Mertens and R. Imbihl, Chem. Phys. Lett. 242, 221 (1995).

${ }^{28}$ A. Makeev and R. Imbihl, J. Chem. Phys. 113, 3854 (2000).

${ }^{29}$ Y. De Decker, H. Marbach, M. Hinz, S. Günther, M. Kiskinova, A. S. Mikhailov, and R. Imbihl, Phys. Rev. Lett. 92, 198305 (2004). 